\title{
Giant duodenal polyp
}

\author{
M. Belhassine', G. Beniuga ${ }^{2}$, M. Henin ${ }^{3}$, X. Chapaux ${ }^{3}$, S. Negrin Dastis ${ }^{1}$, Ph. Warzee ${ }^{1}$
}

(1) Department of Gastroenterology and Hepatology, GHDC, Charleroi, Belgium; (2) Institute of Pathology and genetics (IPG), Charleroi, Belgium; (3) Department of Radiology, GHDC, Charleroi, Belgium.

\section{Question}

A 52 year-old man was referred with a history of anemia and fatigue. There was no melena and he was not taking any medication

Blood analysis revealed microcytic anemia, with a hemoglobin of 9,7 g/dl (NR 13-18), MCV of 65,8 fl (NR 80-100) and serum ferritine was below $5 \mathrm{ng} / \mathrm{ml}$ (NR 30-400) with normal coagulation. There was no inflammation, no renal insufficiency and liver function tests were normal. Vitamine B12 and folic acid were normal.

He underwent endoscopic work-up. Colonoscopy was normal. Gastroscopy revealed a large pedunculated polyp $(5 \times 2 \mathrm{~cm})$ localized in the duodenal bulb and it was eroded on the top (Figure 1A and B). Multiple biopsies were taken showing no abnomarlity. There was no Helicobacter pylori nor metaplasia.

An abdominal computed tomography was performed and confirmed the duodenal polyp without signs of locoregional invasion (Figure 1C).

What is the diagnosis? What would you recommend?

Finally, it was decided to remove the polyp endoscopically using a diathermic snare loop, after having compressed the stalk with clips and an endoloop to prevent bleeding.

A polyp of 4,8X2,2 cm was extracted (Figure $2 \mathrm{~A}$ and B) and histopathological analysis revealed Brunner's gland hyperplasia without any sign of malignancy (Figure 2C).

Follow-up gastroscopy performed two months later did not show recurrence and anemia was corrected. Brunner's gland hyperplasia corresponds to a submucosal benign hamartomatous lesion that is frequently observed in the duodenum.

The etiology remains unclear (1). Chronic infection with Helicobacter pylori, chronic gastritis and duodenitis may be related to this pathology (2).

When the hamartoma size exceeds $2 \mathrm{~cm}$, it is more frequently associated with gastrointestinal bleeding, obstructive symptoms, pancreatitis and very rarely invasive carcinoma (3).

In the current case, endoscopic removal was indicated to confirm the diagnosis, to exclude underlying malignancy and to treat iron-deficiency anemia.

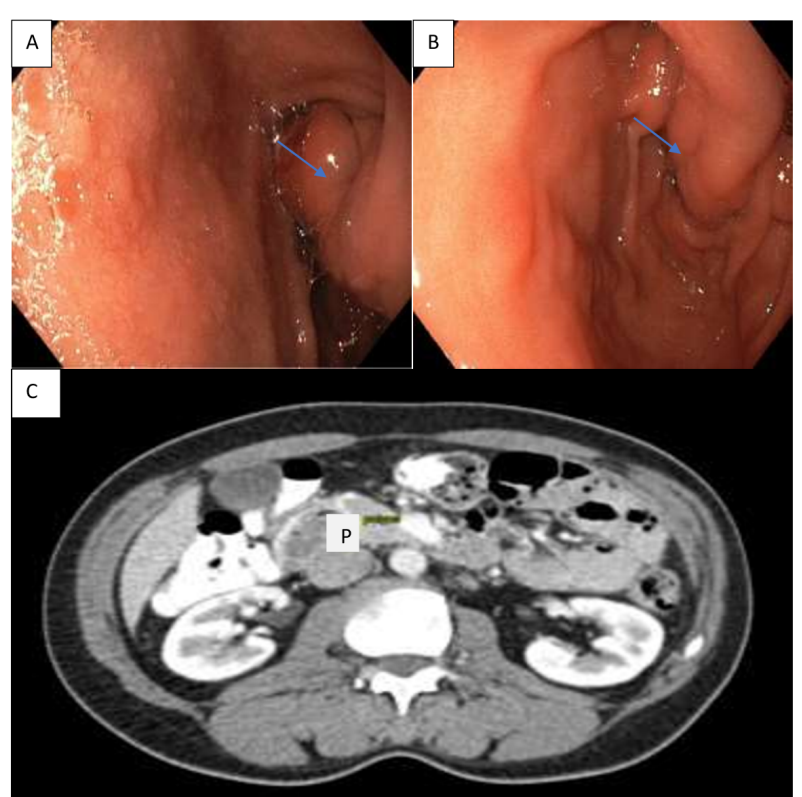

Figure 1. - Upper endoscopy (duodenal bulb). Giant duodenal polyp (arrow on the top of the polyp) (A). Upper endoscopy (gastric cavity). Polyp's large peduncle swinging in the gastric part (arrow on the peduncle) (B). Abdominal computed tomography. Giant duodenal polyp without any sign of local invasion (P letter) $(\mathrm{C})$.

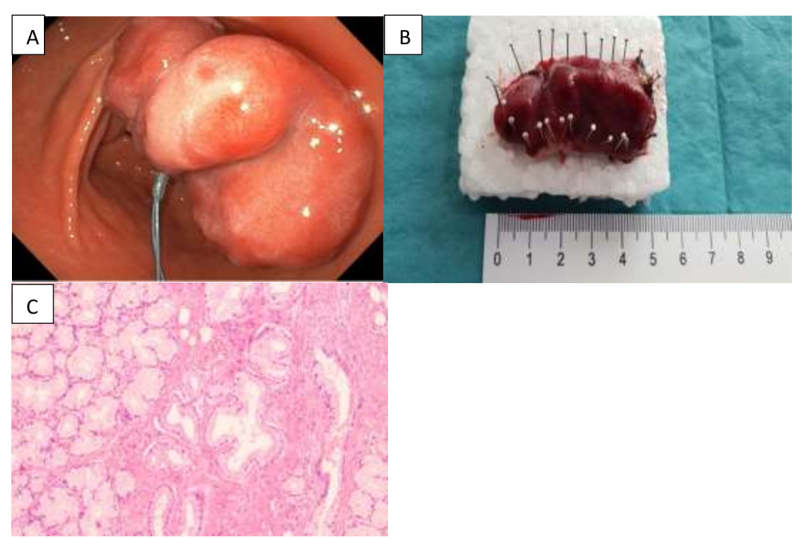

Figure 2. - Upper endoscopy (gastric cavity). Polyp's resection using endoloop.(A). Picture of the resected polyp. Measured using a ruler (B). Histological image using hematoxylin and esosin staining of tissue with a X5 magnification. Monomorphic aspect of the glands without cytonuclear atypia (C).

\footnotetext{
Correspondence to: Maia Belhassine, Department of Gastroenterology and Hepatology Medicine, GHDC, Charleroi, Belgium.

Email: maia.belhassine@student.uclouvain.be

Submission date : 13/04/2020

Acceptance date : 15/12/2020
} 


\section{References}

1. C. TOUSSAINT, L. LiBbRECHT, H. DANO, H. PIESSEVAUX. Endoscopic features, pathological correlates and possible origin of foveolar gastric metaplasia presenting as a duodenal polyp. Acta Gastroenterol Belg 2019, 82: 257-260.
2. T. SAKURAI, H. SAKASHITA, G. HONJO, I. KASYU, T. MANABE. Gastric foveolar metaplasia with dysplastic changes in Brunner gland hyperplasia: possible precursor lesions for Brunner gland adenocarcinoma. Am J Surg Pathol, 2005, 29: 1442-1448.

3. E. STERMER, N. ELIAS, D KEREN, T. RAINIS, O GOLDSTEIN, A. LAVY. Acute pancreatitis and upper gastrointestinal bleeding as presenting symptoms of a duodenal Brunner's gland hamartoma. Can. J. Gastroenterol, 2006, 20: 541-54. 